\title{
Patrice NICOLAS
}

Institut National de la Santé et de la Recherche Médicale

Lab. Groupe de Recherches U.84

Hôpital de la Salpêtrière

75634 PARIS CEDEX 13

ABSTRACT

The discrepancy between potential distributions recorded on the scalp and on the cerebral cortex, as a result of the electric field propagation through the surrounding shells, requires development of techniques which enable us to compute one distribution as a function of the other. The method presented is based on a physical model consistent with neurophysiological patterns, and in which the sources (encephalitic charges and currents) are multipolar distributions. The general field equations, applied to a convenient closed domain, lead to a family of Helmholtz equations which govern the transmission of the potential pseudo-periodic components. Local boundary conditions having been imposed by the multipolar assumption, the solutions are deduced from an elementary source system. It is then shown that the distribution of the surface potential (on the scalp) is obtained by convolution of the cortical potential distribution and a transfer function which is a solution of the Helmholtz equation associated with an elementary source system. An approximation of the cortical distribution can be computed by discrete deconvolution. Finally, various simulation experiments on digital computer allowed us to test the model, by comparison with empirical data. 


\section{INTRODUCTION}

The potential changes dealt with clinical and experimental neurophysiology are with few exceptions recorded from skin electrodes which are placed at a distance from the active cells; therefore, if we want to draw conclusions as to the generators of the potential, we come upon many problems due to the propagation of the field through various tissues. In spite of experimental investigation, the interpretation of brain activities recorded on the scalp is particularly problematic, because they result from numerous sources which are very heterogeneous. More precisely, by setting electrodes on the scalp, one tries to have information about the cortical sites of abnormal signals, as a consequence of pathological processes. It would be then more usefull to implant the captors in the brain; for obvious reasons, we cannot often use such an optimal procedure on man. Hence, the best thing we could try to obtain is an approximation of the cortical surface potential, proceeding from scalp records. For this purpose, we present a general model which can be used both for theoretical explanation and experimental test.

\section{SETTING THE PROBLEM}

The physical system under consideration can be described as follows:

The encephalon is an unknown set of electrical sources, varying both in space and time, which occupy a closed domain whose boundary is the cortical surface $S$. The neural generators induce two potential distributions, $V_{S}$ on $S$ and $V_{S}$, on the scalp surface S', by propagation through several layers of media like rachidian cephalitic liquid (RCL), dura matter, skull and skin (the brain's shells). We have then to exhibit the relationship of $\mathrm{V}_{\mathrm{S}}$ and $\mathrm{V}_{\mathrm{S}}$, (fig. 1 ).

As a good approach, it is possible to consider each media as being linear, homogeneous and isotropic. Locally, we can also assure that the thicknesses $h_{i}$ of the layers vary little in space; moreover, the radius of curvature of the various surfaces is always 'large' with respect to the distance $h=\sum h_{i}$ which separates $S$ from $S$ '. Figure 1 summarizes the local geometry of the physical system just described.

\section{GENERAL EQUATIONS}

The potential $V$ is a pseudo-random function (Smith \& Schadé, 1970), which is continuous everywhere and can be expressed as the resultant of a finite number of pseudo-periodic components:

$$
v(x, t)=\sum_{n=1}^{N} U_{n}(x) \operatorname{Exp}\left(j f_{n}(t)\right)
$$

The space functions $U_{n}$ are assumed to be continuous on $R^{3}$. The phases $f_{n}$ are stricly in- 


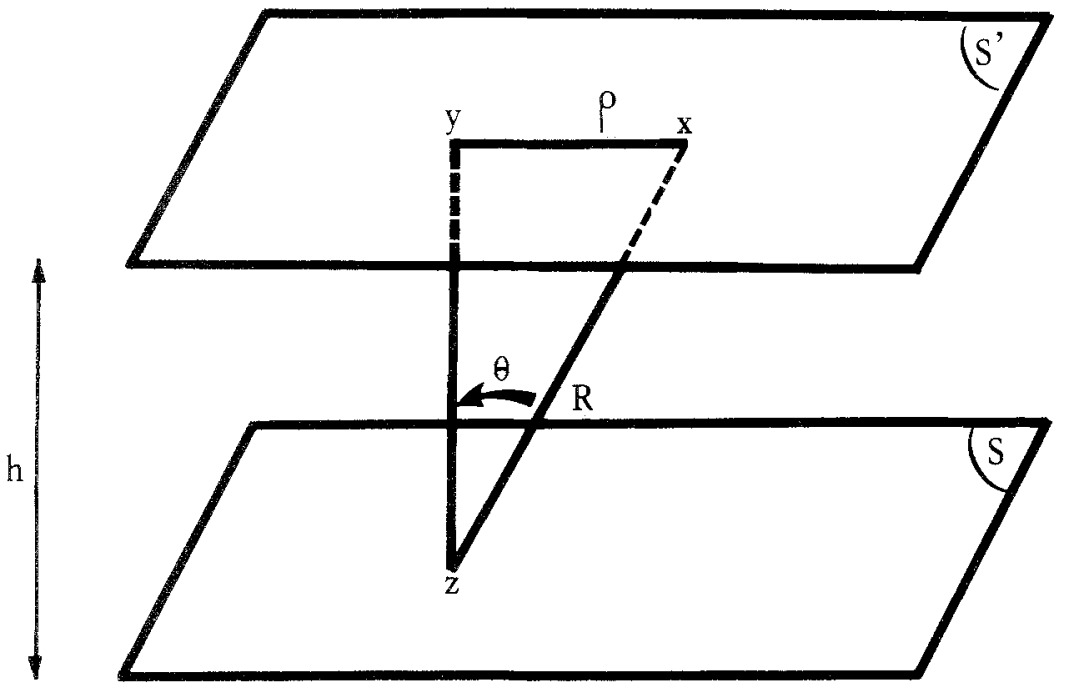

FIGURE 1.- Local geometry of the physical problem.

creasing and $\mathrm{C}^{1}$ on every time interval; hence, their first derivatives are uniformly bounded. Moreover, within every domain which does contain no source, the propagation of the potential is governed by the following equation (Panofsky \& Phillips, 1955):

$$
\Delta V-\epsilon \mu \frac{\partial^{2} V}{\partial t^{2}}-\mu \gamma \frac{\partial V}{\partial t}=0
$$

where the coefficients are characteristics of the media such as:

$$
\varepsilon \mu \sim \frac{1}{c^{2}}
$$

According to this latter estimate, and if we take into account the low frequency range under consideration, then we can neglect the second derivative with respect to time, and we obtain:

$$
\Delta V-\mu \gamma \frac{\partial V}{\partial t}=0
$$

Thus, the propagation of the bio-potential between cortex and scalp is a diffusion process, and more precisely a Wiener process (Dynkin, 1965).

Let $k_{n}$ be now an time-dependant function such as: 


$$
\mathbf{k}_{\mathbf{n}}^{2}(t)=-j \mu \gamma \frac{\mathbf{d f}}{\mathrm{dt}}
$$

It follows from (1) and the equation (3) that we have then:

$$
\Delta U_{n}+k_{n}^{2} U_{n}=0
$$

for each spatial component $U_{n}$ of $V$. This latter equation is called the Helmholtz equation of the Wiener process (Bouix, 1966). Its general solution is given by the expansion:

$$
\mathrm{U}(\mathrm{r}, \theta, \varphi)=\sum_{\mathrm{n}=0}^{\infty} \sum_{\mathrm{m}=0}^{\mathrm{n}} \mathrm{z}_{\mathrm{n}}(\mathrm{kr})\left(\mathrm{a}_{\mathrm{nm}} \cos \mathrm{m} \varphi+\mathrm{b}_{\mathrm{nm}} \sin \mathrm{m} \varphi\right) \mathrm{P}_{\mathrm{n}}^{\mathrm{m}}(\cos \theta),
$$

where $z_{n}$ and $p_{n}^{m}$ respectively are Bessel functions and Legendre polynomials.

\section{BOUNDARY PROBLEM}

The boundary conditions obviously must be compatible with physiological data, but the only thing we can assume to be known is the potential $V_{S}$, on the cortical surface $S$. Then, it is necessary to make one more assumption, in order to have uniqueness. For this purpose, let us consider an elementary source set; according to the structures of the neural nets, we can assume that the field which is induced by this set is locally characterized as follows:

AT ANY POINT OF THE CORTICAL SURFACE, THE ELECTRICAL IMAGE OF THE UNDERLYING GENERATORS IS IDENTICAL TO THAT OF A VIRTUAL MULTIPOLE VITH CYLINDRICAL SYMMETRY AND AXIS PERPENDICULAR TO THE SURFACE.

Such an hypothesis involves that the boundary conditions are given by a non-uniform distribution of multipolar sources. The cylindrical symetry leads to $m=0, \varphi=0$ in the expansion (6), and we have:

$$
U(r, \theta)=\sum_{n=0}^{\infty} a_{n} z_{n}(k r) P_{n}(\cos \theta)
$$

Moreover, if the elementary source sets are $2^{q}$ - poles, then we have:

$$
\mathbf{a}_{\mathbf{n}}=0, \quad \mathbf{n}=0,1, \ldots, \mathbf{q}-1
$$

and:

$$
\sum_{n=q+1}^{\infty} a_{n} z_{n} p_{n}=o\left(\frac{1}{k r}\right)
$$

In other words, the main part of the expansion ( $\left.6^{\prime}\right)$ is given by the only q-th term. Here a first optimization problem arises, which is to find the optimal q for the best approxima- 
tion; it is still unresolved, because of the lack of convenient experimental data. Until now, the neurophysiologist only was interested in dipolar sheet models, or even dipoles deep within the brain (Bremer, 1949; Eccles, 1951; Bishop \& Clare, 1952; Calvet et al., 1964, Geisler \& Gerstein, 1961). Nevertheless, such a modelization seems to be much too simple with regard to the complexity of the neural networks, because thousands of depolarization currents spread over each cortical neuron at the same time. Therefore, a multipaLar sheet hypothesis appears more realistic, at least from a theoretical point of view.

Whatever $q$ may be, we know that $z_{q} \mathrm{p}_{\mathrm{q}}$ is rapidly decreasing as a function of the distance. If we take into account the background noise, the multipolar potential $V$ approximately vanishes beyond a cylinder $C$, whoses bases are disks $D$, $D^{\prime}$ on $S$ and $S^{\prime}$. Let $V_{D}$ be the zero-order trace of $V$, restricted in $D$. Then, for one multipole, we have the following boundary problem:

$$
\left\{\begin{array}{l}
\text { To find } V \text { such as: } V \in C^{\circ}(\bar{C}) \text { and: } \\
\text { (i) } \Delta V+k^{2} V=0 \text { inside } C \\
\text { (ii) }\left.V\right|_{D}=V_{D} \text { on } D
\end{array}\right.
$$

In this formulation, (i) stands for the propagation and (ii) expresses the choice of partial boundary conditions. As $V$ must be continuous everywere, it is easy to show that the above problem is well-posed.

Now, let $x$ be a running point on $S$, and $y$ any point on $S^{\prime}$; according to the multipolar assumption, we have then:

$$
V_{D},(y)=V_{D}(x) z_{q}\left(\frac{k h}{u(\rho)}\right) P_{q}(u(\rho)),
$$

where:

$$
u(\rho)=\cos \left(\operatorname{Arctg} \frac{\rho}{h}\right), \text { and } \rho=|x-y| \text {. }
$$

If $g=z_{q} P$, wh an obvious change of variables we finally obtain:

$$
\mathrm{V}_{\mathrm{D}^{\prime}}(\mathrm{y})=\mathrm{V}_{\mathrm{D}}(\mathrm{x}) \mathrm{g}(\mathrm{x}-\mathrm{y})
$$

Hence, if we consider the resl potential distribution $V_{S}$ which is induced on $S^{\prime}$ by the whole set of cortical sources, then we have the following approximation:

$$
\mathrm{V}_{\mathrm{S}^{,}}(\mathrm{y})=\int_{\mathrm{D}} \mathrm{V}_{\mathrm{S}}(\mathrm{x}) \mathrm{g}(\mathrm{x}-\mathrm{y}) \mathrm{dx},
$$

where $V_{S}$ is the real potential distribution on $S$, As $g$ is approximately vanishing beyond the disk $D$ which is centered on $y$, we can only consider its restriction $g_{D}$ in $D$. Then we can write:

$$
\mathrm{V}_{\mathrm{S}^{\prime}}=\mathrm{V}_{\mathrm{S}^{*}} \mathrm{~g}_{\mathrm{D}^{\circ}}
$$


Stated another way, through an obvious isomorphism between $D$ and $D^{\prime}$, we come upon a convolution. As a matter of fact, $g_{\mathrm{D}}$ depends on $\mathrm{y}$ through the parameter $\mathrm{h}$ and the function $\mathrm{k}$, because the shells are not strictly uniform. Nevertheless, if we assume (as we have since the outset) that there exists a 'large enough' domain where the gradient of $h$ and $k$ is negligible, then $g_{D}$ is unvarying by translation in such a domain. Hence, $g_{D}$ will be called the transfer function of the potential, in the sense of the multipolar hypothesis. The following statement sumnarizes the results:

LET $V_{S}$ BE A PSEUDO-PERIODIC POTENTIAL DISTRIBUTION ON THE CORTICAL SURFACE S. IF $v_{S}$ OBEYS THE LOCAL MULTIPOLAR ASSUMPPION, THEN THE POTENTIAL DISTRIBUTION $V_{S}$, WHICH IS INDUCED ON THE SCALP IS APPROXIMATED BY THE CONVOLUTION EQUATION (12).

This statement is true for pseudo-periodic potential; for any pseudo-random potential as defined in $\$$ III, we obtain analogous results by linearity.

Furthermore, there are weak eddy currents inside the shells, which do not come from the encephalitic generators. Hence, to the potential induced by these generators, one must add some background noises which are represented in the boundary problem by a convenient function $\mathrm{F}(\mathrm{x}, \mathrm{t})$ on the right-hand side of the equation (i). We have taken it into account by choosing $F$ as uniform random noise for further computing.

\section{NUMERICAL ESTIMATES}

In order to test the model with empirical data, some numerical estimates have been made, such as attenuation, vision field of the electrodes, and geometrical approximation error. For this purpose, the dipolar hypothesis has been used, because it was obviously both the most simple and the best known.

\section{1) Attenuation}

The attenuation effect is measured by the transfer function, where $q=1$. Two factors are involved: frequency and distance. The first one can be considered as negligible, because the potential changes under consideration are much too slow. The shell's thickness dependance, on the contraxy, have the greatest importance; for its estimate, we have computed $g$ for five different $h$ values. The result is a set of curves which express the attenuation of the potential as a function of the distance from a dipole, for each thickness $h$. More precisely, let $E$ be an electrode (pin-point electrode) situated at the poirt $x$ on the scalp, and let $z$ be any point of the cortical surface, projected onto $y$ on the scalp ( $f i g .1$ ). The attenuation of a signal recorded by $\mathrm{E}$ from $z$ is given by:

$$
10 \log _{10}(g(x-y))
$$

This function increases (in absolute value) both with the distance $\rho$ from the dipole axis and the total thickness $h$ of the shells. Figure 2 shows the main variations of this atte- 


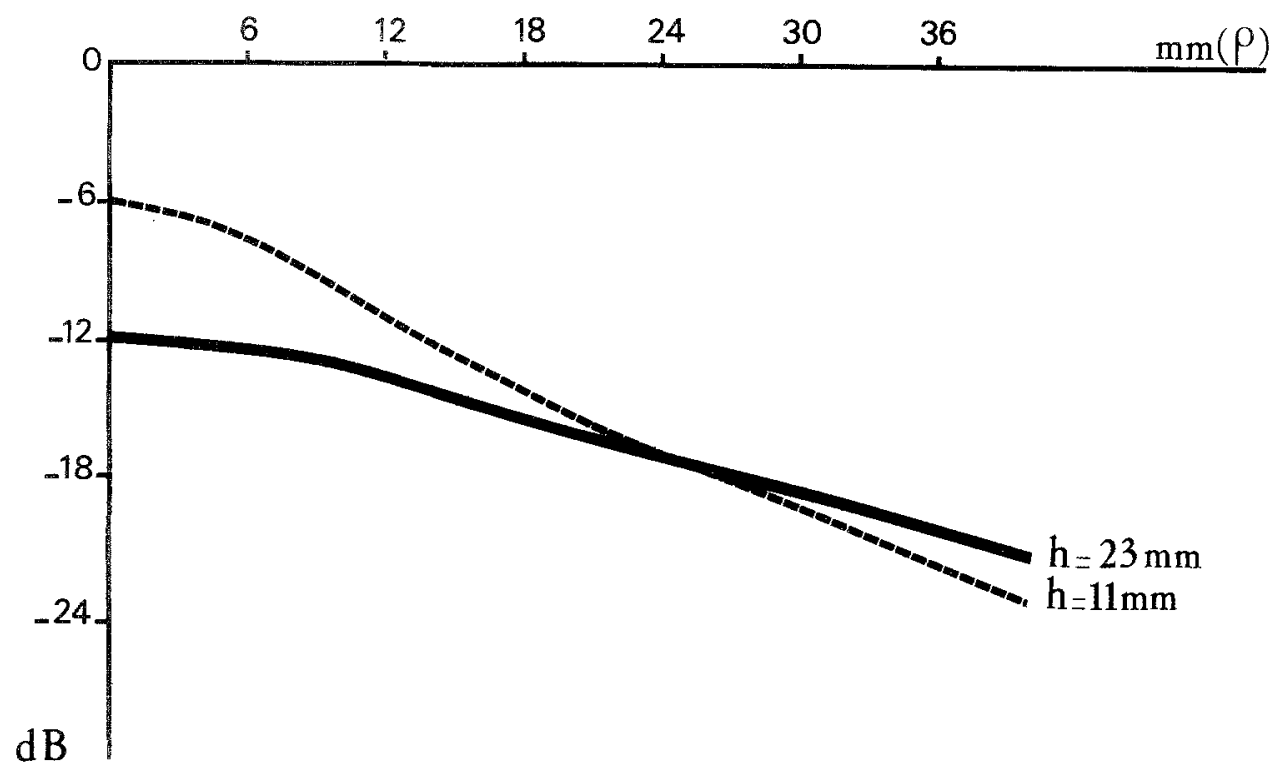

FIGURE 2.- Attenuation curves, as functions of the distance from a dipole axis (elementary source set). The dotted curve corresponds to the minimum thickness of the layers between cortex and scalp; the solid one corresponds to the maximum thickness of these layers; the middle curves (not plotted) have the same intersection as those in the figure.

nuation. All the curves are cutting each other close to $-16 \mathrm{~dB}$. Stated another way, the attenuation is superior to $16 \mathrm{~dB}$ beyond a disk of $24 \mathrm{~mm}$ radius which is centered over the emission point on the cortex. As this is true whatever $h$ may be, then we can estimate that the vision field of a scalp electrode has the same radius, because the ratio signal/noise is always superior to $15 \mathrm{~dB}$.

\section{2) Geometrical error}

The boundary problen is only defined in a 'rather small' cylindrical domain $C$, lying between scalp and cortex. The attenuation estimates allow us to stipulate the real dimensions of this cylinder; they also permit us to compute the error which is introduced by approximating locally the surfaces with their tangent planes. It follows from the preceding that the contribution of $D^{\prime}$ s center (on the cortex) to the potential on the lateral boundary of $\mathrm{C}$ is zero. Then, let us assume that the radius of curvature of the skull is about $9.5 \mathrm{~cm}$ in the zone under consideration (admissible mean value in the parietal zone). Under this condition, the average error on the attenuation is less than .3 dB; the error on the radius of $D$ is at the most $.25 \mathrm{~mm}$. The geometrical approximation of the model is thus admissible, at least for 'rather regular zones' of the skull. 


\section{SIMULATION EXPERIMENTS}

The model's behaviour has been tested on digital computer, for various cortical distributions, by the means of simulation experiments. For this purpose, the folloving simulated device has been used:

On the scalp, inside a circular zone of $24 \mathrm{~mm}$ radius, are assumed to be placed 49 electrodes, one of them central. They have a circular section of $4 \mathrm{~mm}$ in diameter, and occupy the nodes of a square grid having links of $6.14 \mathrm{~mm}$. Any two electrodes are thus always at a minimum distance of $2.14 \mathrm{~mm}$ from each other. All recordings are monopolar with average reference. On the cortex, a similar montage is assumed to be set up inside a concentric circular zone of radius $48 \mathrm{~mm}$. Thus, in order to take into account the preceding estimates, the simulation is restricted to a domain which is equivalent to a 'spot of vision' or visual field for scalp electrodes. In the same way, their diameter corresponds to a value in common use with real experiments. Figure 3 roughly schematizes the device such simulated.

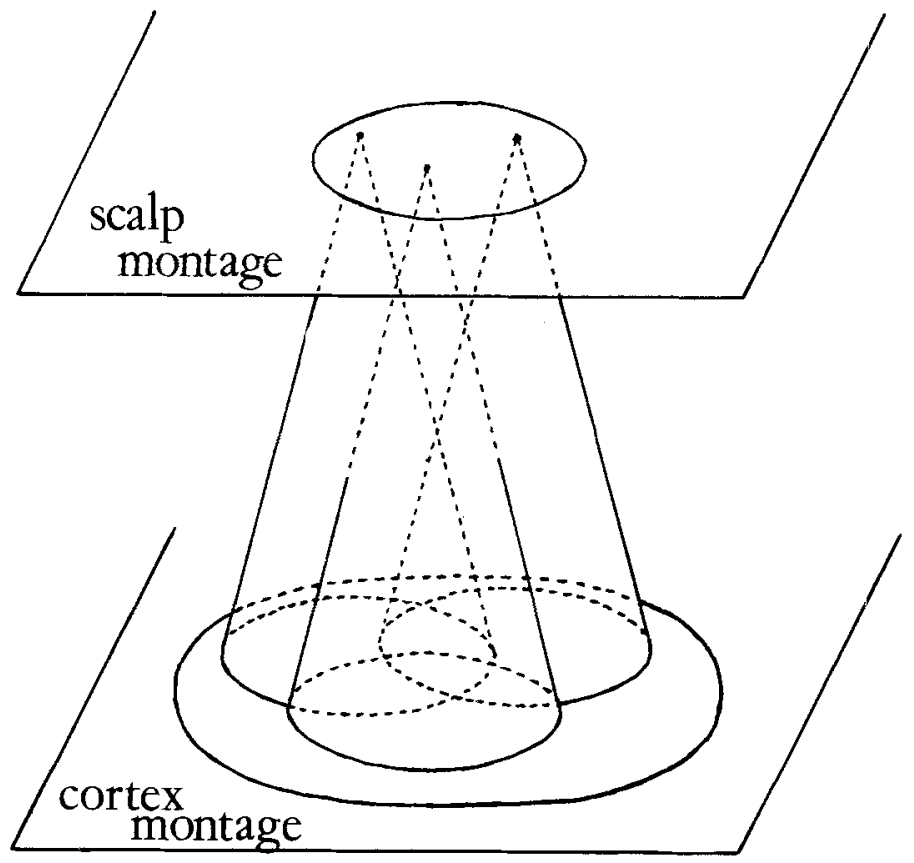

FIGURE 3.- Simulated device: each skin electrode has a fiel of vision which is a circular cortical area of $48 \mathrm{~mm}$ in diameter.

Furthermore, we assume that the cortical area under consideration holds up three mixed pseudo-periodic activities: ALPHA waves (10 - $15 \mathrm{~Hz})$, BETA waves (20 - 25 
$\mathrm{Hz}$ ) and THETA waves $(3-7 \mathrm{~Hz})$. The spatial densities of these components are respectively choosen as elliptic, exponential and parabolic distributions.

Figure 4 shows traces corresponding with two recordings from central electrodes (one of them on the cortex and the other on the scalp), during a pive seconds interval for a cortical distribution having a weak stationnary gradient. These traces show almost the same types of waveshapes; nevertheless, various patterns (lettered) are rather badly reproduced, reversed or even undetected on the scalp. The average attenuation reaches $-4 \mathrm{~dB}$, but the smoothing out is negligible. Such an example is a 'good case', where two superimposed electrodes receive similar signals. This is due to the regularity of the cortical distribution, whose components are spread out with minor divergences of density in the whole area which is explored.

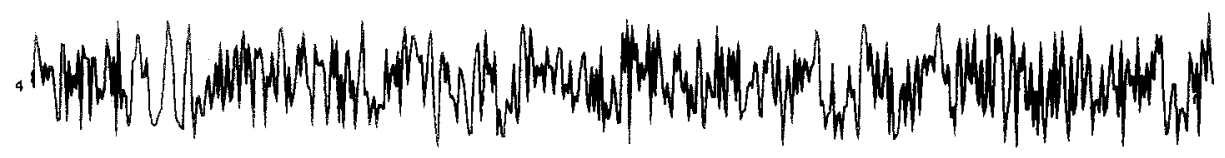

A $\quad$ B $\quad C \quad$ D $\quad$ E F
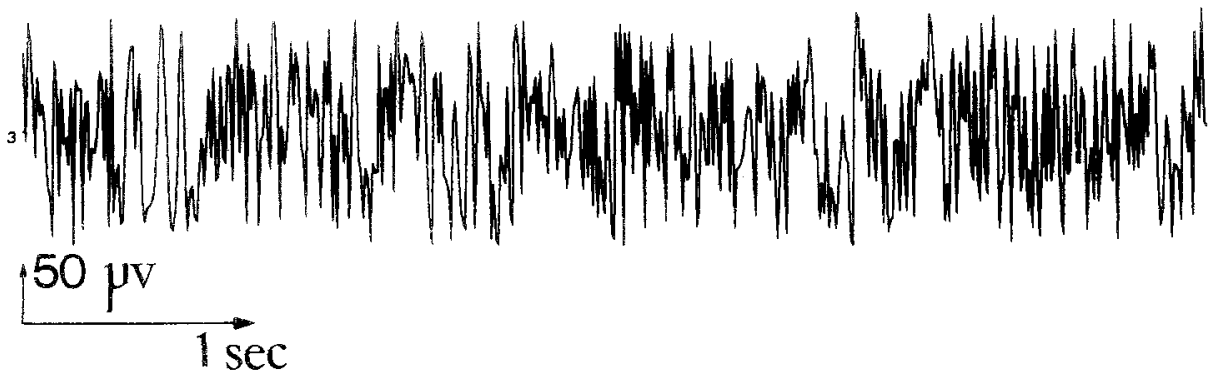

PIGURE 4.- Transmission of a weak gradient complex activity. At the top: scalp recording; at the bottom: cortical recording. Letters point out zones of discrepancy. Time constant: $10 \mathrm{msec}$.

On the contrary, the traces of figure 5 show the effect of a strong gradient cortical distribution. In this simulated experiment, an ample BETA rhytm is localized in a $5 \mathrm{sq}$. cm cortical area which is centered on the electrodes axis; a weak ALPHA wave is spread out the whole 'spot' which is seen by the skin electrode. Under this condition, there is a total discrepancy between the two recordings: the ALPHA waveshape is detected 
on the scalp, whereas the only BETA rhytm does exist inside the underlying cortical zone.
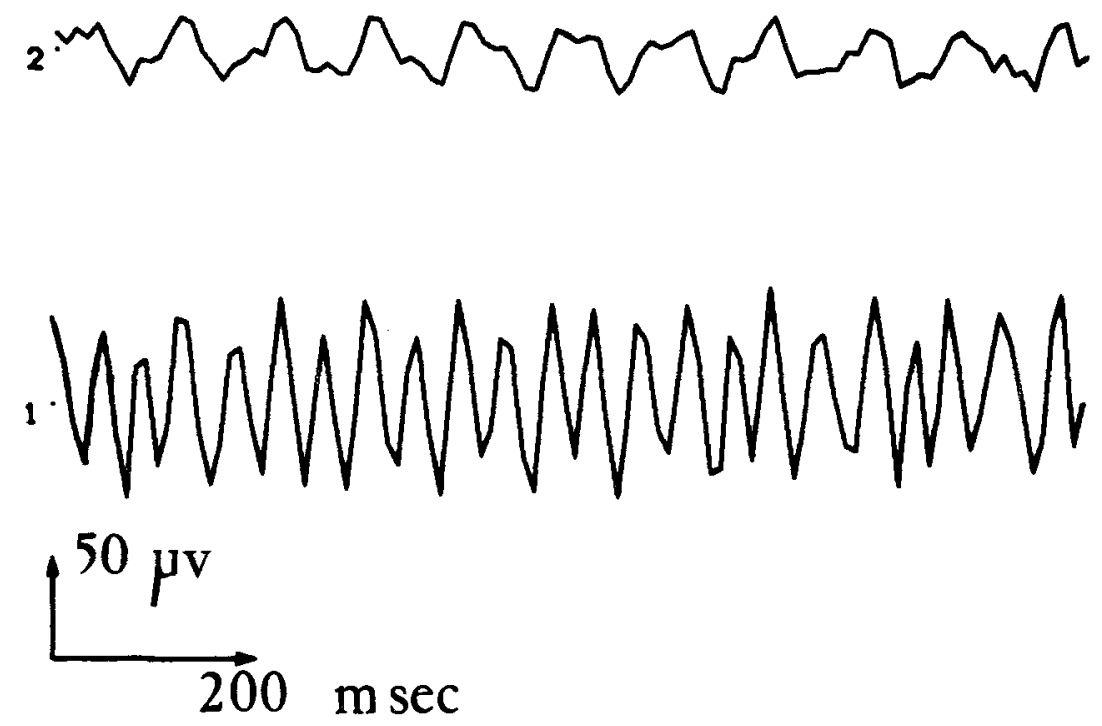

FIGURE 5.- Transmission of a complex activity having a strong space gradient. At the top: the scalp recording; at the bottom: the cortical recording. There is a total discrepancy between the levels.

In figure 6 we give the distributions of surface potential, for fixed time, inside the two homologous 'spots' on cortex and scalp. Here we see that the maximum BETA and THETA activity completely disappears from the scalp. Moreover, the extension of negative potentials is larger on the scalp, and it tends to the middle of the disk, whereas the amplitudes are stronger in the upper left quadrant. This latter effect is due to large amplitude potentials beyond the boundary of the disk which is shown in figure 6 . In the main, however, the gradient is weaker on the scalp, and there is a spatial smoothing of the distributions.

\section{Remark}

The above simulated traces do not aim at being realistic; more precise$1 y$, they are not intended to simulate real traces, and their only purpose is helping us to see in what way various shapened activities can be transmitted from cortex to scalp, in the sense of the model. 


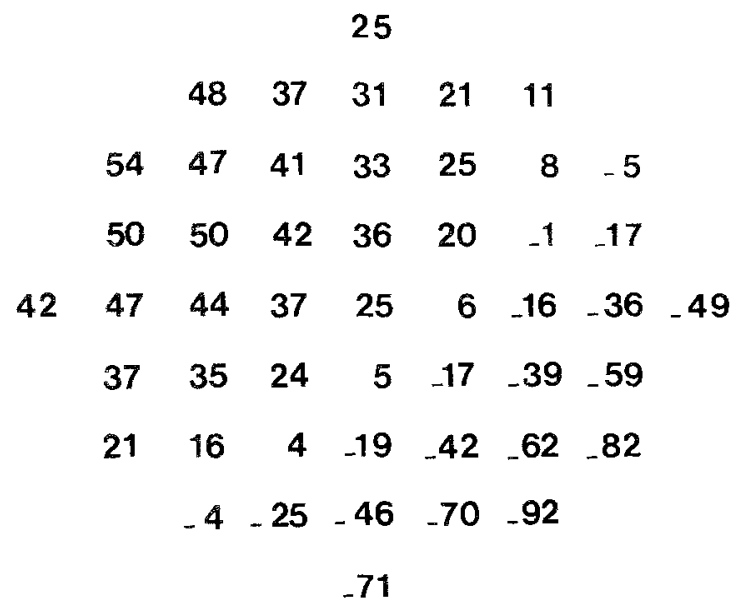

16

$\begin{array}{lllll}11 & 21 & 19 & 11 & -1\end{array}$

$\begin{array}{lllllll}28 & 23 & 39 & 47 & 46 & 13 & -11\end{array}$

$\begin{array}{lllllll}25 & 41 & 58 & 68 & 71 & 30 & -16\end{array}$

$\begin{array}{lllllllll}23 & 34 & 58 & 64 & 48 & 47 & 9 & -32 & -44\end{array}$

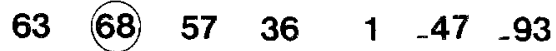

$\begin{array}{lllllll}37 & 45 & 13 & -26 & -53 & -69 & -76\end{array}$

$\begin{array}{lllll}14 & -25 & -55 & -69 & -78\end{array}$

$-69$

FIGURE 6.- Potential distributions inside homologous 'spots' of 48 mm in diameter. At the top: the scalp distribution; at the bottom: the cortical distribution. The potential values are expressed in microvolts. The circle and the rhomb respectively mark the THE'PA and BETA naximum activities on the cortex level; they are not detected on the scalp. 
The results of the simulation essentially arise from the well-known properties of convolution. Indeed, the integration tends to smooth over the strong gradient images, while better spread components remain detectable, even if they are absent from the sub-jacent cortical area. A small amplitude activity can be transmitted if it occupies a sufficiently large cortical zone, while other, although of far greater amplitude, are not seen on the scalp. These results are tallied with the empirical observations. Moreover, it is easy to see that any multipolar assumption would provide results of the same type, within numerical deviations. This tolerance reinforces the generality of the model, but limits the span of the classical hypotheses made on the cortical sources patterns.

The convolution defines $V_{S}$, as a function of $V_{S}$ and $g$. In practice, for clinical applications, the question is rather how to compute $\mathrm{V}_{\mathrm{S}}$ on the cortex, using the values of $\mathrm{V}_{\mathrm{S}}$, which are picked up on the scalp. In other words, we have to reverse the convolution, and the main problem is to find an optimal method for the best approximation, which takes into account both the small number of captors and the noise level. However, given an appropriate definition of experimental conditions, it seems possible to compute the electrocorticogram as a function of the surface EEG, in the sense of the model, with a sufficient accuracy.

\section{REFERENCES}

BISHOP G.H. \& CLARE M.H.: Sites of origin of electric potential in striate cortex. J. Neurophysiol., 1958, 10:201-220.

BOUIX M.: Les discontinuités du rayonnement électromagnétique. Dunod, Paris, 1966, 241 pp. BREMER F.: Considérations sur l'origine et la nature des "ondes" cérébrales. EEG clin. Neurophysiol., 1949, 1: 177-193.

CALVET J., CALVET M.C. \& SCHERRER J.: Etude stratigraphique de l'activité EEG spontanée. EEG clin. Neurophysiol., 1964, 17: 109-125.

DYNKIN E.B.: Markov Processes. Springer-Verlag, Berlin, 1965, t.1, 357 pp.

ECCLES J.C.: Interpretation of action potentials evoked in the cerebral cortex. EEG clin. Neurophysiol., 1951, 19: 217-229.

GEISLER C.D. \& GERSTEIN G.L.: The surface EEG in relation to its sources. EEG clin. Neurophysiol., 1961, 13: 927-934.

PANOPSKY W.A. \& PHILLIPS M.: Classical electricity and magnetism. Addison-Wesley, Reading, Mass., 1955, $400 \mathrm{pp}$.

SMITH J. \& SCHADE J.P.: Computer Programing for parameter Analysis of the Electroencephalogram. In Computers and Brain. Progress in Brain Research, vol.33, Amsterdam, 1970. 